\title{
RAY SHOOTING AMIDST CONVEX POLYHEDRA AND POLYHEDRAL TERRAINS IN THREE DIMENSIONS*
}

\author{
PANKAJ K. AGARWAL ${ }^{\dagger}$ AND MICHA SHARIR ${ }^{\ddagger}$
}

\begin{abstract}
We consider the problem of ray shooting in a three-dimensional scene consisting of $m$ (possibly intersecting) convex polyhedra or polyhedral terrains with a total of $n$ faces, i.e., we want to preprocess them into a data structure, so that the first intersection point of a query ray and the given polyhedra can be determined quickly. We present a technique that requires $O\left((m n)^{2+\varepsilon}\right)$ preprocessing time and storage, and can answer ray-shooting queries in $O\left(\log ^{2} n\right)$ time. This is a significant improvement over previously known techniques (which require $O\left(n^{4+\varepsilon}\right)$ space and preprocessing) if $m$ is much smaller than $n$, which is often the case in practice. Next, we present a variant of the technique that requires $O\left(n^{1+\varepsilon}\right)$ space and preprocessing, and answers queries in time $O\left(m^{1 / 4} n^{1 / 2+\varepsilon}\right)$, again a significant improvement over previous techniques when $m \ll n$.
\end{abstract}

Key words. arrangements, ray shooting, range searching, data structures, parametric search, random sampling

AMS subject classifications. 52B11, 68P05, 68Q20, 68Q25

1. Introduction. The ray-shooting problem can be defined as follows: Given a collection $\Gamma$ of $n$ objects in $\mathbb{R}^{d}$, preprocess $\Gamma$ into a data structure so that one can quickly determine the first object of $\Gamma$ intersected by a query ray.

The ray-shooting problem has received much attention in the last few years because of its applications in computer graphics and other geometric problems [1], [3], [4], [5], [6], [9], [10], [14], [17], [21], [28]. Most of the work to date studies the planar case, where $\Gamma$ is a collection of line segments in $\mathbb{R}^{2}$. Chazelle and Guibas proposed an optimal algorithm for the special case where $\Gamma$ is the boundary of a simple polygon [17]. Their algorithm answers a ray-shooting query in $O(\log n)$ time using $O(n)$ space; simpler algorithms, with the same asymptotic performance bounds, were recently developed in [14] and [22]. If $\Gamma$ is a collection of arbitrary segments in the plane, the best-known algorithm answers a ray-shooting query in time $O\left(\frac{n}{\sqrt{s}} \log ^{O(1)} n\right)$ using $O\left(s^{1+\varepsilon}\right)$ space and preprocessing ${ }^{1}$ [1], [6], [9], where $s$ is a parameter that varies between $n$ and $n^{2}$. Although no lower bound is known for this case, it is conjectured that this bound is close to optimal. In spite of some recent developments, the three-dimensional ray-shooting problem seems much harder and it is still far from being fully solved. The general three-dimensional ray-shooting problem is to preprocess a collection of $n$ triangles, so that the first triangle hit by a query ray can be computed efficiently. If the triangles are the faces of a convex polyhedron, then an optimal algorithm, with $O(\log n)$ query time and linear space, can be obtained using the hierarchical decomposition scheme of Dobkin and Kirkpatrick [20]. If the triangles form a polyhedral terrain (a piecewise-linear surface intersecting every vertical line in ex-

${ }^{*}$ Received by the editors February 16, 1993; accepted for publication (in revised form) July 22, 1994. A preliminary version of this paper appeared in Proc. 4th ACM-SIAM Symp. on Discrete Algorithms, 1993, pp. $260-270$.

${ }^{\dagger}$ Department of Computer Science, P.O. Box 90129, Duke University, Durham, NC 27708-0129. This work was supported by National Science Foundation grant CCR-91-06514.

${ }^{\ddagger}$ School of Mathematics, Tel Aviv University, Tel Aviv 69978, Israel, and Courant Institute of Mathematical Sciences, New York University, New York, NY 10012. This work was supported by National Science Foundation grant CCR-91-22103 and by grants from the U.S.-Israeli Binational Science Foundation, the G.I.F. (the GermanIsraeli Foundation for Scientific Research and Development), and the Fund for Basic Research administered by the Israeli Academy of Sciences.

${ }^{1}$ Throughout this paper, bounds of this kind mean that, given any arbitrarily small positive constant $\varepsilon$, the algorithm can be fine-tuned so that its performance satisfies the bound; the multiplicative constants in such bounds usually depend on $\varepsilon$ and tend to $\infty$ as $\varepsilon \downarrow 0$. 
actly one point), then the technique of Chazelle et al. [15] yields an algorithm that requires $O\left(n^{2+\varepsilon}\right)$ space and answers ray-shooting queries in $O(\log n)$ time. Nontrivial solutions to the general problem were obtained only recently; see [4], [6], and [10] for some of these results. The best-known algorithm for ray shooting among triangles in three dimensions is due to Agarwal and Matoušek [5]; it answers a ray-shooting query in time $O\left(\frac{n^{1+\varepsilon}}{s^{1 / 4}}\right)$ after $O\left(s^{1+\varepsilon}\right)$ space and preprocessing. The parameter $s$ can range between $n$ and $n^{4}$. If $s$ assumes its maximum value, queries can be answered in $O(\log n)$ time; see [5], [6], and [28] for more details. We remark that no nontrivial lower bounds are known for the three-dimensional problem as well, although such bounds are known for the related simplex range-searching problem [12], which is used as a subprocedure in the solutions just mentioned.

The performance of these algorithms is rather inefficient when $n$ is large, so a natural objective is to find special cases where this performance can be improved. The case that we consider here is where the three-dimensional scene is formed by $m$ convex polyhedra or polyhedral terrains with a total of $n$ faces (general nonconvex polyhedra can be decomposed into convex pieces and be replaced by these pieces). In many typical instances of the problem $m$ is much smaller than $n$; for example, curved objects, like balls, cylinders, cones, etc., are usually approximated by a polyhedron with a large number of faces. Our goal is to develop an algorithm whose performance depends on both $m$ and $n$, and is much better than that of the general technique when $m \ll n$.

In this paper we achieve this goal, presenting a technique that uses $O\left((m n)^{2+\varepsilon}\right)$ storage and answers ray-shooting queries in $O\left(\log ^{2} n\right)$ time. Our algorithm is the first algorithm for ray shooting among convex polyhedra (or polyhedral terrains) whose performance depends on both $m$ and $n$ and matches the performance of [5] when $m \approx n$. We also present another algorithm that answers a query in time $O\left(m^{1 / 4} n^{1 / 2+\varepsilon}\right)$ using $O\left(n^{1+\varepsilon}\right)$ space and preprocessing, so it matches the bound of [5] when $m \approx n$, but is considerably faster when $m \ll n$.

In [7] we have presented an algorithm to preprocess a collection of $m$ convex polygons in the plane, with a total of $n$ vertices, into a data structure of size $O(m n \log m)$, so that a ray-shooting query can be answered in $O\left(\log ^{2} n \log ^{2} m\right)$ time. If the polygons are disjoint, or the starting point of the ray always lies in the common exterior of the polygons, then the space and preprocessing can be improved to $O\left(\left(m^{2}+n\right) \log m\right)$. The algorithm works even for a collection of disjoint simple polygons.

A problem related to ray shooting among a collection of convex polyhedra in three dimensions is the so-called stabbing problem, where one wants to determine whether a query line intersects all polyhedra. This problem seems to be easier than the ray-shooting problem: Pellegrini and Shor [29] have described a data structure of size $O\left(n^{2+\varepsilon}\right)$ that can answer a stabbing query in $O(\log n)$ time.

We will first describe, in $\S 2$, the overall structure of the algorithm. We next present, in $\S 3$, an algorithm for detecting an intersection between a query segment and a collection of convex polyhedra or polyhedral terrains, which is the main subroutine used in our algorithm. For the sake of convenience, we describe the algorithm only for a collection of convex polyhedra, but the same technique works for polyhedral terrains as well. Next, in $\S 4$, we develop a variant of the technique for answering a query efficiently if only close-to-linear space is allowed. In $\S 5$, we give an application of our results to translational motion planning in $\mathbb{R}^{3}$ : given $m$ convex polyhedral obstacles, with a total of $n$ faces, and a polyhedral object $B$, with $k$ vertices, free to translate amidst them, we show how to preprocess them in time and space $O\left((\mathrm{kmn})^{2+\varepsilon}\right)$, so that, given any free placement $z$ of $B$ and direction $\mathbf{u}$, we can compute in time $O\left(\log ^{2} k n\right)$ the first obstacle to be hit as $B$ is translated from $z$ in direction $\mathbf{u}$. Again, this is a substantial improvement over previous results when $m \ll k n$. We conclude in $\S 6$ with a discussion of our results and a few open problems. 
2. The overall algorithm. Let $\mathcal{P}=\left\{P_{1}, \ldots, P_{m}\right\}$ be a set of $m$ convex polyhedra, let $n_{i}$ be the number of edges in $P_{i}$, and put $n=\sum_{i=1}^{m} n_{i}$ (we prefer to have $n$ denote the total number of edges, rather than the number of faces, of the $P_{i}$ 's; by Euler's relation, these two quantities differ only by a small multiplicative factor). Without loss of generality assume that each face of $P_{i}$ is triangulated; otherwise we can triangulate all faces of $P_{i}$ by adding $O\left(n_{i}\right)$ additional edges. For the sake of convenience, we split the boundary of each $P_{i}$ into its top portion (visible from $z=+\infty$ ) and its bottom portion (visible from $z=-\infty$ ). We construct separate data structures for the top portions and for the bottom portions, and answer a ray-shooting query by searching in both structures and by selecting the output point nearest to the ray origin. In what follows we describe only the data structure for the top portions of the given polyhedra; with a slight abuse of notation, we will refer to these top portions also as "polyhedra." We note that this step is not required if the $P_{i}$ 's are polyhedral terrains.

Our general ray-shooting scheme is based on the parametric searching technique of Agarwal and Matoušek [4]. In this technique we build a data structure for solving segmentintersection detection queries, each asking whether a query segment $e$ intersects any of the (top portions of the) given polyhedra. Given a query ray $\rho$, we replace it by the segment $a w$, where $a$ is the origin of $\rho$ and $w$ is the first point of intersection between $\rho$ and the given polyhedra. We query the data structure with the segment $a w$. Of course, we do not know $w$ (our goal is to find it!), so we feed our data structure with a generic, unspecified input $w$. As we will see below, each step of the algorithm asks a question of the following form: given a query point $p$ and a hyperplane $h$, determine whether $p$ lies above, below, or on $h$; here $p$ is either the origin $a$ of $\rho$, the Plücker point of the line containing $\rho$, or the generic point $w$. In the first two cases, we can answer the question in $O(1)$ time. To determine the position of $w$ with respect to a plane $h$, it is sufficient to determine whether $a$ and $w$ lie on the same side of $h$. We compute the intersection point $\sigma$ of $\rho$ and $h$. If $\rho$ does not intersect $h$, we can immediately conclude that $a$ and $w$ lie on the same side of $h$. Otherwise, we invoke the segment-intersection detection procedure with the segment $a \sigma$. If $a \sigma$ intersects any of the polyhedra in $\mathcal{P}$, then $a$ and $w$ lie on the same side of $h$; otherwise they lie on the opposite sides of $h$. This also restricts the allowed range of $w$. When the algorithm terminates, we obtain the exact location of $w$, thereby answering the original ray-shooting query. It is shown in [4] that the performance of this parametric searching technique is only slightly worse (by a logarithmic factor) than the cost of a single (explicit) segment-intersection detection query; see [4] for more details.

3. Segment-intersection detection. We now present an algorithm for the segment-intersection detection problem, i.e., preprocess $\mathcal{P}$ into a data structure, so that one can quickly determine whether a query segment intersects any of the polyhedra in $\mathcal{P}$. In this section we aim to achieve fast (polylogarithmic) query time, at the expense of storage and preprocessing. The opposite case, that of using only close-to-linear storage at the expense of query time, will be studied in $\S 4$. We will construct two data structures. The first one, denoted as $\Psi_{1}(\mathcal{P})$, determines whether $e$ intersects a face of some $P_{i}$ whose $x y$-projection does not contain any of the endpoints of the $x y$-projection of $e$. The second data structure, denoted as $\Psi_{2}(\mathcal{P})$, determines whether $e$ intersects a face of some $P_{i}$ whose $x y$-projection contains one of the endpoints of the $x y$-projection of $e$. Throughout this section we will use $\gamma^{*}$ to denote the $x y$-projection of an object $\gamma$ in $\mathbb{R}^{3}$, and $A^{*}$ to denote $\left\{\gamma^{*} \mid \gamma \in A\right\}$ for a set $A$ of such objects.

3.1. First data structure. In this subsection we describe a data structure $\Psi_{1}(\mathcal{P})$ that determines whether the query segment intersects a face of some $P_{i}$ whose projection does not contain any endpoint of $e^{*}$. Let $E_{i}$ denote the set of edges of $P_{i}$. We project them onto the $x y$-plane, and let $E_{i}^{*}$ denote the set of resulting projected segments. Let $E^{*}=\bigcup_{i=1}^{m} E_{i}^{*}$. We 

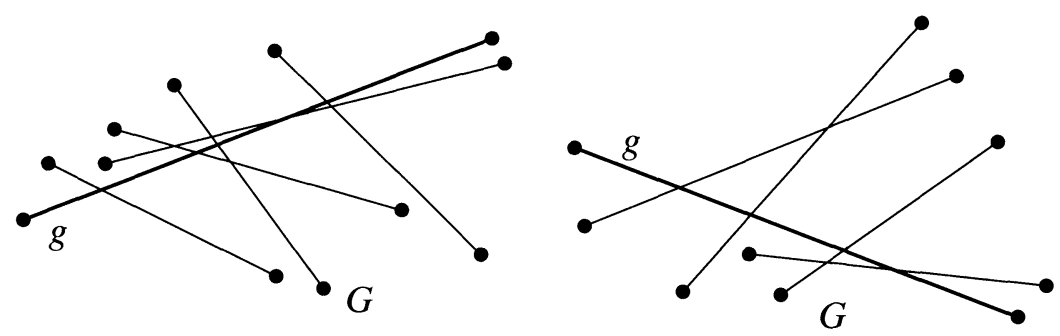

FIG. 1. A canonical subset $G$ of the output for a query segment $g$; (i) $g$ intersects all segments of $G$ from below; (ii) $g$ intersects all segments of $G$ from above.

preprocess $E^{*}$ into a data structure of size $O\left(n^{2+\varepsilon}\right)$, using a variant of the technique described in Agarwal and Sharir [6], so that the set of all segments of $E^{*}$ intersected by a query segment in the $x y$-plane can be represented as $O(\log n)$ pairwise disjoint precomputed subsets. The algorithm of [6] constructs a multilevel partition tree on $E^{*}$. Roughly speaking, it stores a family of subsets of $E^{*}$, called canonical subsets, into a tree-like data structure. There are at most $O\left(\left(n / 2^{j}\right)^{2+\varepsilon}\right)$ canonical subsets of size between $2^{j-1}$ and $2^{j}$. For a given query segment $g$, the canonical subsets that form the query output can be computed in $O(\log n)$ time, and there is a constant number of output subsets of size between $2^{j-1}$ and $2^{j}$ for each $j=0,1, \ldots$ Furthermore, for each canonical subset $G$ of the query output, either the left endpoints of all segments in $G$ lie above the line containing the query segment $g$, or all of them lie below that line. In the first case $g$, considered as a rightward-directed segment, intersects all of these segments from below, and in the second case it intersects all of them from above. (In the $x y$-plane, a rightward-directed segment $g$ is said to intersect another segment $e$ from "below" if they intersect and the left endpoint of $e$ lies above the line containing $g$; intersection from "above" is defined symmetrically; see Fig. 1.) See [4] and [6] for details. In what follows we only consider the case where $g$ intersects all segments of $G$ from below.

Let $\Gamma^{*}$ be a canonical subset of $E^{*}$, and let $\Gamma$ be the set of corresponding pohyhedra edges, and put $v=\left|\Gamma^{*}\right|$. Let $\Gamma_{i}^{*}=\Gamma^{*} \cap E_{i}^{*}$, and let $\mu \leq v$ denote the number of nonempty $\Gamma_{i}^{*}$ 's. Set $s=\lceil\nu / \mu\rceil$. We orient the edges of $\Gamma^{*}$ from left to right. We preprocess $\Gamma$ so that, for a (directed) query line $\ell$ in $\mathbb{R}^{3}$ whose $x y$-projection intersects all segments of $\Gamma^{*}$, one can quickly determine whether $\ell$ passes above or below the edges in $\Gamma$. The way in which we have oriented the $x y$-projections of the edges in $\Gamma$ and of $\ell$ ensures that the above/below relationships between $\ell$ and these edges are determined solely by the sign of the relative orientations between $\ell$ and the lines containing these edges. This will enable us to determine whether a query segment intersects any of the given polyhedra, as will be described in more detail below. The relative orientation of two oriented lines $\ell, \lambda$ in $\mathbb{R}^{3}$ is defined to be the orientation of any simplex $a b c d$, where $a, b \in \ell, c, d \in \lambda$, so that $\ell$ is oriented from $a$ to $b$ and $\lambda$ is oriented from $c$ to $d$. Equivalently, it is also the sign of the inner product between the two vectors in projective 5-space representing the Plücker coordinates of the two lines. (For the sake of convenience, we will not distinguish between the projective 5-space and the affine 5 -space $\mathbb{R}^{5}$.) To be more precise, $\ell$ can be mapped to a point $\pi(\ell)$, called a Plücker point, and $\lambda$ can be mapped to a hyperplane $\varpi(\lambda)$, called a Plücker hyperplane, in $\mathbb{R}^{5}$, so that $\ell$ has positive orientation with respect to $\lambda$ if and only if $\pi(\ell)$ lies above the hyperplane $\varpi(\lambda)$. The Plücker points of all lines in $\mathbb{R}^{3}$ lie on a quadric surface, known as the Plücker surface, in $\mathbb{R}^{5}$. More details concerning Plücker's coordinates and relative orientations can be found in [15] and [31].

Recall that we are only preprocessing the upper portions of the polyhedra, which implies that the (relative) interiors of edges of $\Gamma_{i}^{*}$ are pairwise disjoint. We define a linear ordering for 
a set $G$ of $t$ nonintersecting segments in the $x y$-plane: let $e, e^{\prime}$ be two segments in $G$; $e \prec e^{\prime}$ if the $x$-projections of $e$ and $e^{\prime}$ overlap and $e^{\prime}$ lies above $e$ along a vertical line (parallel to the $y$-axis), if the $x$-projections of $e$ and $e^{\prime}$ are disjoint and $e$ lies to the left of $e^{\prime}$. This is indeed a linear ordering, and it can be computed in $O(t \log t)$ time, as shown by Guibas, Overmars, and Sharir [21]. They have also shown that, for any $e, e^{\prime} \in G$, if $e \prec e^{\prime}$ and a rightward-directed line intersects both of them from below, then it intersects $e$ before $e^{\prime}$.

We sort each $\Gamma_{i}^{*}$ according to this ordering and, abusing the notation slightly, we denote the resulting sequence also by $\Gamma_{i}^{*}$. Suppose $\Gamma_{i}^{*}=\left(e_{0}^{*}, e_{1}^{*}, \ldots, e_{t_{i}}^{*}\right)$. We mark the first, the last, and every $s$ th edge of $\Gamma_{i}^{*}$, i.e., we mark $e_{0}^{*}, e_{s}^{*}, e_{2 s}^{*}, \ldots, e_{t_{i}}^{*}$. For each marked edge $e_{s j}^{*}$, let $\Gamma\left(e_{s j}\right)=\left\{e_{s j+1}, e_{s j+2}, \ldots, e_{u}\right\}$, where $u=\min \left\{t_{i},(s+1) j\right\}$, be the set of edges of $P_{i}$ whose $x y$-projections form the block of edges of $\Gamma_{i}^{*}$ following $e_{s j}^{*}$ and ending at the next marked edge. Let $G$ be the set of edges of polyhedra in $\mathcal{P}$ corresponding to the marked segments of $\Gamma^{*}$, each oriented so that its $x y$-projection is rightward directed; note that

$$
|G| \leq \sum_{i}\left(\left\lceil\frac{t_{i}}{s}\right\rceil+1\right) \leq \sum_{i}\left(\frac{t_{i}}{s}+2\right) \leq \frac{\mu}{v}\left(\sum_{i} t_{i}\right)+2 \mu \leq 3 \mu .
$$

We will construct on $G$ a data structure based on a partitioning scheme due to Chazelle, Sharir, and Welzl [18]. For a segment $\gamma \in \mathbb{R}^{3}$, this structure decomposes $G$ further into canonical subsets, so that, for each canonical subset of $G$, either all the corresponding original polyhedra edges lie above $\gamma$ or all of them lie below $\gamma$. Next, for each canonical subset $Q$ in the output, we determine whether there is an edge in some $\Gamma(e)$, for $e \in Q$, that passes on the other side of $\gamma$, thereby implying that $\gamma$ intersects the polyhedron $P_{i}$ containing $e$. In more detail, this is done as follows.

Map the (directed) line containing each segment of $G$ to its Plücker hyperplane in $\mathbb{R}^{5}$; let $H$ denote the set of resulting hyperplanes, and put $t=|H| \leq 3 \mu$. Set $r$ to be some sufficiently large constant. We compute a $(1 / r)$-net $R$ of $H$ of size $O(r \log r)$. (We call a subset $R \subseteq H$ a $(1 / r)$-net if every (relatively open) simplex intersecting more than $|H| / r$ hyperplanes of $H$ intersects a hyperplane of $R$; it is well known that there exists such an $R$ with the prescribed size.) $R$ can be computed in $O(t)$ time if $r$ is constant [11], [23]. We triangulate the arrangement $\mathcal{A}(R)$. Let $\Xi$ denote the simplices of the triangulation that intersect the Plücker surface. By a result of Aronov, Pellegrini, and Sharir [8], the number of simplices in $\Xi$ is $O\left(r^{4} \log ^{5} r\right)$. By construction, each simplex in $\Xi$ intersects at most $t / r$ hyperplanes of $H$.

For each $\Delta \in \Xi$, let $H_{\Delta} \subseteq H$ denote the set of hyperplanes that intersect the interior of $\Delta$. We also associate with $\Delta$ two other subsets $U_{\Delta}$ and $L_{\Delta}$ of $H ; U_{\Delta}$ is the set of hyperplanes that lie fully above $\Delta$, and $L_{\Delta}$ is the set of hyperplanes that lie fully below $\Delta$.

We construct two auxiliary data structures on $U_{\Delta}$ and $L_{\Delta}$. Let

$$
\begin{aligned}
& \bar{U}_{\Delta}=\bigcup\left\{\Gamma(e) \mid e \text { is an edge corresponding to a hyperplane in } U_{\Delta}\right\}, \\
& \bar{L}_{\Delta}=\bigcup\left\{\Gamma(e) \mid e \text { is an edge corresponding to a hyperplane in } L_{\Delta}\right\} .
\end{aligned}
$$

Note that $\left|\bar{U}_{\Delta}\right|,\left|\bar{L}_{\Delta}\right| \leq s t$. We map the lines containing the edges of $\bar{U}_{\Delta}$ to their Plücker hyperplanes in $\mathbb{R}^{5}$ and preprocess their lower envelope for point-location queries, using an algorithm of Clarkson [19]. That is, we preprocess the hyperplanes into a data structure, so that we can quickly determine whether a query point lies below all hyperplanes. Similarly, we map the edges of $\bar{L}_{\Delta}$ to their Plücker hyperplanes and preprocess their upper envelope for similar point location queries. Each point location structure requires $O\left((s t)^{2+\delta}\right)$ space and preprocessing time, for any $\delta>0$, and answers a query in $O(\log s t)$ time; see [19] for details.

Our data structure needs one more ingredient: for each simplex $\Delta \in \Xi$ and for each polyhedron $P_{i}$ we consider the first (marked) edge, if any, of $\Gamma_{i}$ that contributes a hyperplane 
to $U_{\Delta} \cup L_{\Delta}$. For each such edge $e$, let $f_{1}, f_{2}$ be the two faces of $P_{i}$ incident to $e$ (if $e$ is the first or the last edge of $\Gamma_{i}$, it is possible that only one of these faces is defined). Let $F_{\Delta}$ be the collection of at most $2 t$ resulting faces. We preprocess $F_{\Delta}$ for segment-intersection detection queries using the techniques described in [5] and [6]. It requires $O\left(t^{4+\delta}\right)$ space and preprocessing time, for any $\delta>0$, and can determine in $O(\log t)$ time whether a query segment intersects any triangle in $F_{\Delta}$.

Finally, we recursively preprocess $H_{\Delta}$, for each simplex $\Delta \in \Xi$. The recursion stops when $\left|H_{\Delta}\right|$ falls below some specified constant $n_{0}$. The resulting structure is a tree $T=T(\Gamma)$ of depth $O(\log n)$, each of whose nodes has degree at most $O\left(r^{4} \log ^{5} r\right)$. We repeat the same procedure for all canonical subsets $\Gamma$ of $E$. This completes the description of the data structure.

Let us analyze the space and preprocessing time of the above structure. First let us analyze the space required by the tree structure $T(\Gamma)$ constructed on a canonical subset $\Gamma$. Let $S(u)$ denote the space required by the subtree of $T$ constructed on a set $H_{\Delta}$ consisting of $u$ hyperplanes. Since the degree of each node in $T$ is $O\left(r^{4} \log ^{5} r\right)$, and the auxiliary structure stored at each child requires $O\left(s^{2+\delta} u^{2+\delta}+u^{4+\delta}\right)$ space, we get the following recurrence:

$$
S(u) \leq \begin{cases}O(1) & \text { if } u \leq n_{0}, \\ c_{1} r^{4} \log ^{5} r \cdot S\left(\frac{u}{r}\right)+c_{2}\left(s^{2+\delta} u^{2+\delta}+u^{4+\delta}\right) & \text { if } u>n_{0},\end{cases}
$$

where $c_{1}, c_{2}$, and $n_{0}$ are some appropriate absolute constants. The solution of this recurrence is

$$
S(u) \leq A s^{2+\delta^{\prime}} u^{4+\delta^{\prime}}
$$

for another $\delta^{\prime}>0$ that tends to 0 with $\delta$ and for some sufficiently large constant $A=A\left(\delta^{\prime}\right)$. Indeed, arguing inductively, (1) obviously holds for $u \leq n_{0}$, and for $u>n_{0}$ we have

$$
\begin{aligned}
S(u) & \leq c_{1} r^{4} \log ^{5} r \cdot S\left(\frac{u}{r}\right)+c_{2}\left(s^{2+\delta} u^{2+\delta}+u^{4+\delta}\right) \\
& \leq c_{1} r^{4} \log ^{5} r A s^{2+\delta^{\prime}}\left(\frac{u}{r}\right)^{4+\delta^{\prime}}+c_{2}\left(s^{2+\delta} u^{2+\delta}+u^{4+\delta}\right) \\
& =A s^{2+\delta^{\prime}} u^{4+\delta^{\prime}}\left(\frac{c_{1} \log ^{5} r}{r^{\delta^{\prime}}}+\frac{c_{2}}{A}\left(\frac{(s u)^{\delta-\delta^{\prime}}}{u^{2}}+\frac{u^{\delta-\delta^{\prime}}}{s^{2+\delta^{\prime}}}\right)\right) \\
& \leq A s^{2+\delta^{\prime}} u^{4+\delta^{\prime}}
\end{aligned}
$$

provided that $\delta^{\prime}>\delta$ and $r, A$ are sufficiently large. Hence, the total space required by $T$ is

$$
S(t)=O\left(s^{2+\delta^{\prime}} t^{4+\delta^{\prime}}\right)=O\left(\lceil\nu / \mu\rceil^{2+\delta^{\prime}} \mu^{4+\delta^{\prime}}\right)=O\left(\nu^{2+\delta^{\prime}} \mu^{2+\delta^{\prime}}\right),
$$

because $\mu \leq \nu$. We calibrate $\delta$ so that $\delta^{\prime}$ is equal to the original $\varepsilon$. Since there are $O\left(\left(n / 2^{j}\right)^{2+\varepsilon}\right)$ canonical subsets of $E^{*}$ of size between $2^{j-1}$ and $2^{j}$, the overall space required by the data structure is

$$
\sum_{j=1}^{\lceil\log n\rceil} O\left(\left(\frac{n}{2^{j}}\right)^{2+\varepsilon}\right) \cdot O\left(2^{j(2+\varepsilon)} m^{2+\varepsilon}\right)=O\left((m n)^{2+\varepsilon^{\prime}}\right),
$$

for another $\varepsilon^{\prime}>0$ that tends to 0 with $\varepsilon$. Hence the total storage required by the data structure is $O\left((m n)^{2+\varepsilon}\right)$ for any $\varepsilon>0$. Following a similar analysis, one can show that the preprocessing time is also $O\left((m n)^{2+\varepsilon}\right)$. 
3.2. Second data structure. Next, we describe the second data structure $\Psi_{2}(\mathcal{P})$, which, given a query segment $\gamma$, determines whether there is a face $f$ of some polyhedron $P_{i}$ such that $\gamma$ intersects $f$ and that $f^{*}$ contains one of the endpoints of $\gamma^{*}$. The data structure is again based on the partitioning scheme due to Chazelle et al. [18]. As in the preceding subsection, we consider here only the top portions of the given polyhedra. Choosing a sufficiently large constant parameter $r$, we partition the plane, in $O(n)$ time, into a collection $\Delta$ of $O\left(r^{2}\right)$ triangles, so that each triangle intersects at most $n / r$ edges of $E^{*}$ [23]. With each triangle $\Delta \in \Delta$, we associate a subset $E_{\Delta}^{*} \subseteq E^{*}$, and a subset $F_{\Delta}$ of faces of polyhedra in $\mathcal{P}$. An edge $e^{*}$ belongs to $E_{\Delta}^{*}$ if the boundary of one of the triangles incident to $e^{*}$ (i.e., the projected polyhedra faces) intersects $\Delta$. A face $f$ belongs to $F_{\Delta}$ if $\Delta \subset f^{*}$. It is easily seen that $\left|E_{\Delta}^{*}\right| \leq 5 n / r$ and $\left|F_{\Delta}\right| \leq m$. We preprocess the triangles of $F_{\Delta}$ for segment-intersection detection queries, as in the first data structure [5], [6]. This structure requires $O\left(m^{4+\delta}\right)$ space and preprocessing time, for any $\delta>0$, and answers a query in $O(\log m)=O(\log n)$ time. We recursively preprocess each $E_{\Delta}^{*}$ and its associated collection of incident faces, and thereby obtain the entire structure. Note that each recursive processing of a set $E_{\Delta}^{*}$ involves $n_{\Delta}=O(n / r)$ edges, at most $2 n_{\Delta}$ incident faces, and $m_{\Delta} \leq n_{\Delta}$ polyhedra to which these edges and faces belong. $\Psi_{2}(\mathcal{P})$ is thus a tree of height $O(\log n)$.

If we denote by $S^{\prime}(m, n)$ the maximum space required by $\Psi_{2}(\mathcal{P})$ for a collection of $m$ polyhedra with $n$ edges, then $S^{\prime}(m, n)$ satisfies the following recurrence:

$$
S^{\prime}(m, n) \leq \begin{cases}O(1) & \text { if } n \leq n_{0}, \\ \sum_{j=1}^{c_{1} r^{2}} S^{\prime}\left(m_{\Delta_{j}}, n_{\Delta_{j}}\right)+c_{2} m^{4+\delta} & \text { if } n>n_{0}\end{cases}
$$

where $m_{\Delta_{j}} \leq n_{\Delta_{j}} \leq \frac{5 n}{r}$, for each $j$, and $n_{0}, c_{1}, c_{2}$ are appropriate constants. The solution of this recurrence is

$$
S^{\prime}(m, n) \leq B(m n)^{2+\varepsilon},
$$

for some constant $B$ and another $\varepsilon>0$ that tends to 0 with $\delta$. Indeed,

$$
\begin{aligned}
S^{\prime}(m, n) & \leq \sum_{j=1}^{c_{1} r^{2}} S^{\prime}\left(m_{\Delta_{j}}, n_{\Delta_{j}}\right)+c_{2} m^{4+\delta} \\
& \leq c_{1} r^{2} B\left(\frac{5 m n}{r}\right)^{2+\varepsilon}+c_{2} m^{4+\delta} \\
& \leq B(m n)^{2+\varepsilon}\left(\frac{5^{2+\varepsilon} c_{1}}{r^{\varepsilon}}+\frac{c_{2}}{B} \cdot \frac{m^{\delta-\varepsilon}}{n^{\varepsilon}} \cdot\left(\frac{m}{n}\right)^{2}\right) \\
& \leq B(m n)^{2+\varepsilon},
\end{aligned}
$$

provided that $\varepsilon>\delta$ and $r, B$ are chosen sufficiently large. The last inequality follows from the fact that $m \leq n$. A similar analysis yields the same bound on the preprocessing time needed to construct the structure.

3.3. Answering a query. We now describe how to answer a query. Let $\gamma$ be a query segment in $\mathbb{R}^{3}$, oriented so that its $x y$-projection is rightward directed, and let $\ell$ be the directed line containing $\gamma$. We want to determine whether $\gamma$ intersects any $P_{i}$.

DEFINITION 3.1. A segment $\gamma$ lies above another segment e in $\mathbb{R}^{3}$ if their xy-projections intersect and the vertical line through this intersection meets $\gamma$ at a point higher than the point at which it meets $e$. 


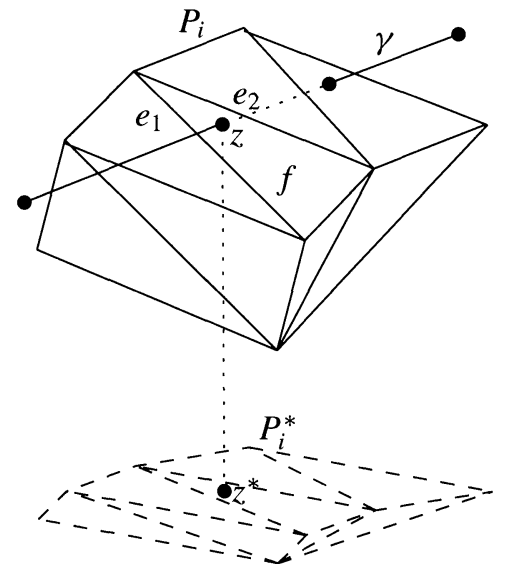

FIG. 2. Illustration of Lemma 3.2.

The query answering procedure is based on the following simple lemma.

LEMMA 3.2. A segment $\gamma=p q$ in $\mathbb{R}^{3}$ intersects a polyhedron $P_{i} \in \mathcal{P}$ if and only if at least one of the following two conditions holds:

(i) A face of $P_{i}$, whose xy-projection contains an endpoint of $\gamma^{*}$, intersects $\gamma$.

(ii) There is a face $f$ of $P_{i}$ such that $\gamma$ lies below (resp., above) one of the edges $e_{1}$ of $f$ and lies above (resp., below) another edge $e_{2}$ of $f$. Moreover, assume that $\gamma^{*}$ intersects $e_{2}^{*}$ after $e_{1}^{*}$; then, for any canonical subset $\Gamma^{*}$ of $E^{*}$ that contains $e_{2}$, either $e_{2}^{*}$ is the first edge in $\Gamma_{i}^{*}$, or there is a marked edge $e^{*}$ in $\Gamma_{i}^{*}$ such that $e_{2} \in \Gamma(e)$, and $\gamma$ lies below (resp., above) $e$ and above (resp., below) $e_{2}$.

Proof. The "if" part is obvious. For the "only if" part, assume that $\gamma$ intersects $P_{i}$ but does not intersect the faces of $P_{i}$ whose projections contain the endpoints of $\gamma^{*}$. Let $z$ be the leftmost intersection point of $P_{i}$ and $\gamma$, and let $f$ be the face of $P_{i}$ containing $z$. Since the endpoints of $\gamma^{*}$ do not lie in $f^{*}, \gamma^{*}$ completely crosses $f^{*}$ (see Fig. 2). Let $e_{1}^{*}$ and $e_{2}^{*}$ be the edges of $f^{*}$ intersected by $\gamma^{*}$ in this order. Since $z$ is the only intersection point of $\gamma$ and $f$, either $e_{1}$ lies below $\gamma$ and $e_{2}$ lies above $\gamma$, or vice-versa.

Assume that $e_{2}$ lies above $\gamma$. Let $\Gamma^{*}$ be a canonical subset of $E^{*}$ containing $e_{2}^{*}$. If $e_{2}^{*}$ is not the first edge in $\Gamma_{i}^{*}$, then let $e^{*}$ be the last marked edge in $\Gamma_{i}^{*}$ immediately preceding $e_{2}^{*}$, i.e., $e_{2} \in \Gamma(e)$. Recall that the first edge of $\Gamma_{i}^{*}$ is marked, so $e^{*}$ is always properly defined. Since $z$ is the leftmost intersection point of $\gamma$ and $P_{i}$, and $e_{2}$ lies above $\gamma$, it is easily seen that $e$ lies below $\gamma$. This completes the proof of the lemma.

In view of the above lemma we can answer a query as follows. First of all, we determine, using $\Psi_{2}(\mathcal{P})$, whether there is a face $f$ of some polyhedron $P_{i}$ such that $\gamma$ intersects $f$ and an endpoint of $\gamma^{*}$ lies in $f^{*}$. If we find such a face, we stop right away. In more detail, we query the structure with the left endpoint $p^{*}$ of $\gamma^{*}$. We follow a path in $\Psi_{2}(\mathcal{P})$ starting from the root. At each node we do the following. If $v$ is a leaf, we determine explicitly whether $\gamma$ intersects any of the faces associated with $v$. Otherwise, let $\Delta$ be the set of triangles associated with $v$. We determine (say, by brute force) the triangle $\Delta \in \Delta$ that contains $p^{*}$. We query the auxiliary structure to determine whether any face of $F_{\Delta}$ intersects $\gamma$. If the answer is yes, we stop. Otherwise we descend to the child of $v$ corresponding to the triangle $\Delta$ and recursively search within $E_{\Delta}^{*}$. We apply the same procedure to the right endpoint of $\gamma$. The correctness of this procedure is easy to verify.

Next, we query $\Psi_{1}(\mathcal{P})$ with $\gamma$. First, we find all segments of $E^{*}$ intersected by $\gamma^{*}$. Let $\Gamma^{*}$ be a canonical subset in the query output. Without loss of generality assume that the left 
endpoints of all segments in $\Gamma^{*}$ lie above $\gamma^{*}$, and all their right endpoints lie below $\gamma^{*}$, so that $\gamma^{*}$ intersects all segments of $\Gamma^{*}$ from below. In this case $\gamma$ lies above (resp., below) a segment $e$ of $\Gamma$ (with both $\gamma^{*}$ and $e^{*}$ being rightward directed) if and only if the relative orientation of $\gamma$ with respect to $e$ is positive (resp., negative); see [15]. By Lemma 3.2, it suffices to determine whether there is a $\Gamma_{i}$ such that either $\gamma$ intersects a face incident to the first marked edge of $\Gamma_{i}$, or $\Gamma_{i}$ contains a marked edge $e$ that lies below (resp., above) $\gamma$ but an edge of $\Gamma(e)$ lies above (resp., below) $\gamma$. We map the (directed) line $\ell$ containing $\gamma$ to its Plücker point $\pi(\ell)$ in $\mathbb{R}^{5}$. We determine the simplex $\Delta \in \Xi$ that contains $\pi(\ell)$ (since $\pi(\ell)$ lies on the Plücker surface, $\Xi$ contains such a simplex $)$. First, we determine in $O(\log n)$ time whether $\gamma$ intersects any triangle of $F_{\Delta}$. If the answer is "yes," we stop right away. Otherwise, we continue as follows.

By construction, $\pi(\ell)$ lies above all hyperplanes of $L_{\Delta}$ and below all hyperplanes of $U_{\Delta}$, and therefore $\gamma$ lies above (resp., below) all edges $e$ corresponding to the hyperplanes of $L_{\Delta}$ (resp., $U_{\Delta}$ ). We now have to determine whether $\gamma$ lies below (resp., above) any edge in $\bar{L}_{\Delta}$ (resp., $\bar{U}_{\Delta}$ ). To do so, we locate $\pi(\ell)$ in the upper envelope of $\bar{L}_{\Delta}$ (resp., in the lower envelope of $\bar{U}_{\Delta}$ ). If $\pi(\ell)$ does not lie above the upper envelope of $\bar{L}_{\Delta}$, then $\pi(\ell)$ lies below some hyperplane of $\bar{L}_{\Delta}$, which implies that $\gamma$ passes below the corresponding edge, and therefore intersects one of the polyhedra. We handle $\bar{U}_{\Delta}$ symmetrically. This completes the description of the query answering procedure. The correctness of the procedure is easy to verify, in view of Lemma 3.2.

Let $Q(u)$ be the maximum query time spent at a subtree of $T$ consisting of $u$ hyperplanes. Since we spend $O(\log n)$ time in querying the auxiliary structure stored at $u$, we get the following recurrence:

$$
Q(u) \leq Q(u / r)+O(\log u) .
$$

The solution of (3) is easily seen to be $O\left(\log ^{2} u\right)$ if $r>1$ is constant, so the total time spent in querying the first data structure, for a fixed canonical set $\Gamma^{*}$ of size $v$, is $O\left(\log ^{2} v\right)$. The solution of (3) can be improved to $O(\log \nu)$ by choosing $r=n^{\varepsilon}$. However, if $r=v^{\varepsilon}$, we cannot use a brute-force method to find the simplex of the partitioning $\Xi$ in Plücker 5space, which contains the query point. Instead, we preprocess the hyperplanes containing the facets of simplices in $\Xi$ for answering point location queries using the algorithm of Chazelle and Friedman [16]. Their algorithm preprocesses a collection of $n$ hyperplanes in $\mathbb{R}^{d}$ into a data structure of size $O\left(n^{d+\delta}\right)$, so that a point location query can be answered in time $O(\log n)$. Thus, the simplex containing a query point can be computed in time $O(\log v)$ using $O\left(\left(r^{4} \log ^{5} r\right)^{5+\delta}\right)=O\left(r^{10+\delta}\right)=O\left(v^{\varepsilon(10+\delta)}\right)$ space, for an arbitrarily small constant $\delta>0$. It is easily seen that this additional structure does not affect the asymptotic bound on the total storage required by $T$. One can similarly modify the algorithm of Agarwal and Sharir [6], so that the overall query time of the first data structure, summed over all canonical subsets of $E^{*}$, also reduces to $O(\log n)$; see [10] and [27] for details. A similar analysis shows that the time spent in querying $\Psi_{2}(\mathcal{P})$ is $O\left(\log ^{2} n\right)$ if $r$ is chosen to be a constant, and that it can be improved to $O(\log n)$ by choosing $r=n^{\varepsilon}$, modifying the structure as above.

Hence, we obtain the following theorem.

THEOREM 3.3. Let $\mathcal{P}=\left\{P_{1}, \ldots, P_{m}\right\}$ be a collection of $m$ (possibly intersecting) convex polyhedra in $\mathbb{R}^{3}$ with a total of $n$ edges. Given any $\varepsilon>0$, we can preprocess $\mathcal{P}$ in time $O\left((m n)^{2+\varepsilon}\right)$ into a data structure of size $O\left((m n)^{2+\varepsilon}\right)$, so that an intersection between $\mathcal{P}$ and a query segment in $\mathbb{R}^{3}$ can be detected in $O(\log n)$ time.

Remark 3.4. Notice that we never used the fact that the $P_{i}$ 's are convex polyhedra. The only property we needed was that the $x y$-projections of edges in each $E_{i}$ were pairwise disjoint (and that the projected faces enclosed by the edges of $E_{i}^{*}$ formed a simply connected 
planar region). Hence, the above algorithm also works for polyhedral terrains. We need the nonintersecting property of $E_{i}^{*}$ to order the segments of a canonical subset $\Gamma$ using the algorithm of Guibas, Overmars, and Sharir [21]. This is the only step that does not extend to arbitrary nonconvex polyhedra. We leave it to the reader to verify that our technique does indeed carry over to the case of polyhedral terrains.

We can now plug the above procedure into the general parametric searching procedure of Agarwal and Matoušek [4]. We have explained in $\S 2$ how this is done. To complete the description, one has to check that the operations that are performed by the above segmentintersection detection procedure conform to the set-up of $\S 2$. In particular, we have to ensure that we can simulate the segment-intersection detection algorithm on the segment $o \sigma$, where $o$ is the starting point of the ray and $\sigma$ is the (unknown) first intersection point of the query ray and $\mathcal{P}$, as described in $\S 2$. First of all, observe that the coordinates of the Plücker point of the line $\ell$ containing a query segment depend only on $\ell$ and not on the endpoints of $\gamma$, so the data structures constructed on Plücker hyperplanes or Plücker points can be searched explicitly, without having to generate any generic comparison, so no oracle calls are required at all. All the other data structures, constructed in two or three dimensions, are searched either with the endpoints of the query segment, or with their $x y$-projections, or with the point dual to the line supporting $\gamma^{*}$. For example, the first level of $\Psi_{2}(\mathcal{P})$ is searched with an endpoint of $\gamma^{*}$, and the first level of the two-dimensional segment-intersection structure is searched with the line supporting $\gamma^{*}$ (actually with the point dual to that line). We leave it to the reader to verify, based on the techniques described in [6] and [5], that each comparison generated by both data structures arises in one of the following tests.

(i) Does a tetrahedron contain an endpoint of $\gamma$ ?

(ii) Does a triangle in the $x y$-plane contain an endpoint of $\gamma^{*}$ ?

(iii) Does the point dual to the line supporting $\gamma^{*}$ lie in a given triangle in the $x y$-plane? Since all of the above questions can be reduced to determining whether a given half-space contains $\sigma$, we can indeed use the general parametric search technique, as described in $\S 2$. We thus obtain the main result of the paper.

THEOREM 3.5. Let $\mathcal{P}=\left\{P_{1}, \ldots, P_{m}\right\}$ be a collection of $m$ (possibly intersecting) convex polyhedra or polyhedral terrains in $\mathbb{R}^{3}$ with a total of $n$ edges. Given any $\varepsilon>0$, we can preprocess $\mathcal{P}$ in time $O\left((m n)^{2+\varepsilon}\right)$ into a data structure of size $O\left((m n)^{2+\varepsilon}\right)$, so that the first intersection point of $\mathcal{P}$ and a query ray can be computed in $O\left(\log ^{2} n\right)$ time.

4. Data structures with almost linear size. In this section we consider the problem of preprocessing $\mathcal{P}$ into a data structure of size $O\left(n^{1+\varepsilon}\right)$, so that a ray-shooting query can be answered in time $O\left(m^{1 / 4} n^{1 / 2+\varepsilon}\right)$. We will use a similar approach to that in the preceding sections, except that we will replace each of the data structures used above by an alternative structure that uses only close-to-linear storage. As above, the structures that we will obtain are multilevel partition trees, composed of rather standard components, but, for the sake of completeness, we will provide a brief description of them. The overall structure of the algorithm is the same as in $\S 3$, that is, the algorithm uses parametric searching to replace ray-shooting queries by segment-intersection detection queries. These queries are handled, on a conceptual level, exactly as above. For the new data structures, we need the following notation.

DEFINITION 4.1. Let $S$ be a set of $n$ points in $\mathbb{R}^{d}$ and let $r<n$ be some parameter. $A$ simplicial $r$-partition for $S$ is a collection $\Pi=\left\{\left(S_{1}, \triangle_{1}\right), \ldots,\left(S_{t}, \triangle_{t}\right)\right\}$, where $S_{1}, \ldots, S_{t}$ form a partition of $S, t \leq 2 r,\left|S_{i}\right| \leq\lceil n / r\rceil$, and $\triangle_{i}$ is a simplex containing $S_{i}$. The maximum number of simplices intersected by a hyperplane is called the crossing number of $\Pi$.

Matoušek has shown that there exists a simplicial $r$-partition with crossing number $O\left(r^{1-1 / d}\right)$ [25], [26]. Agarwal and Matoušek [5] proved that if $S$ lies on an algebraic surface 
of some fixed degree, then the crossing number can be improved to $O\left(r^{1-1 /(d-1)} \log ^{d /(d-1)} r\right)$. Moreover, if $r=O(1)$, such a simplicial partition can be computed in linear time.

4.1. First data structure. We begin by describing the modified version of the first data structure, denoted as $\bar{\Psi}_{1}(\mathcal{P})$. Using the technique of Agarwal and Sharir [6], one can preprocess the set $E$ of polyhedron edges into a data structure of size $O\left(n^{1+\varepsilon}\right)$, consisting of $O\left(\left(n / 2^{j}\right)^{1+\varepsilon}\right)$ canonical subsets of size between $2^{j-1}$ and $2^{j}$, for each $j=0,1, \ldots$, so that all segments of $E^{*}$ intersected by a query segment in the $x y$-plane can be reported as a collection of $O\left(n^{1 / 2+\varepsilon}\right)$ pairwise disjoint canonical subsets; moreover, for each $j$ there are $O\left(\left(n / 2^{j}\right)^{1 / 2+\varepsilon}\right)$ canonical subsets of size between $2^{j-1}$ and $2^{j}$ in the query output. For each canonical subset in the query output, the query segment meets all its edges from below, or meets all of them from above. Let $\Gamma$ be a canonical subset of this level of the data structure. We construct a secondary data structure $T(\Gamma)$ on $\Gamma$, similar to the one in the previous section. Put $v=|\Gamma|$ and define $\mu$ and $s$ as in $\S 3.1$. Let $G$ be the set of marked edges of $\Gamma$ as defined in the previous section. We map the lines containing the segments of $G$ (oriented from left to right) to their Plücker points (rather than hyperplanes as in the previous section) in $\mathbb{R}^{5}$. Let $S$ be the set of resulting points; put $t=|S|$. Let $r$ be some appropriate constant. Since all points of $S$ lie on the (quadratic) Plücker surface, we can construct, as remarked above, a simplicial $r$-partition $\Pi=\left\{\left(S_{1}, \triangle_{1}\right), \ldots,\left(S_{u}, \triangle_{u}\right)\right\}$ of $S$ with crossing number $O\left(r^{3 / 4} \log ^{5 / 4} r\right)$. Let $t_{i}=\left|S_{i}\right|$, for $i=1, \ldots, u$. We construct a secondary structure for each $S_{i}$, of the form described below, and then preprocess each pair $\left(S_{i}, \triangle_{i}\right)$ recursively. The resulting structure is a two-level partition tree of depth $O(\log n)$. Let $E\left(S_{i}\right)$ denote the edges of $E$ corresponding to the points in $S_{i}$, and let

$$
\bar{U}_{i}=\bigcup\left\{\Gamma(e) \mid e \in E\left(S_{i}\right)\right\}
$$

Put $\bar{u}_{i}=\left|\bar{U}_{i}\right| \leq s t_{i}$. We map each line containing an edge of $\bar{U}_{i}$ to its Plücker point in $\mathbb{R}^{5}$ and preprocess the set of resulting points into a linear-size data structure that answers empty half-space queries (given a query half-space $g$ in $\mathbb{R}^{5}$, it determines whether $g$ contains any point of $\left.\bar{U}_{i}\right)$ in time $O\left(\bar{u}_{i}^{1 / 2+\delta}\right)$, for some $0<\delta<\varepsilon$; see [24]. We also preprocess the triangles incident to the edges in $E\left(S_{i}\right)$ for segment-intersection detection queries, using the algorithm of Agarwal and Matoušek [5]; it requires $O\left(t_{i}^{1+\delta}\right)$ space and answers a query in time $O\left(t_{i}^{3 / 4+\delta}\right)$. This completes the description of the first data structure. Since a secondary structure constructed on $u$ points requires $O\left(u^{1+\delta}\right)$ space, the total space required by the first data structure for a fixed canonical subset $\Gamma$ with $v$ edges is $O\left(v^{1+\delta}\right)$. Summing it over all canonical subsets of $\Gamma^{*}$, we obtain

$$
\sum_{j=1}^{\lceil\log n\rceil}\left(\frac{n}{2^{j}}\right)^{1+\varepsilon} \cdot O\left(2^{j(1+\delta)}\right)=O\left(n^{1+\varepsilon}\right) \sum_{j=1}^{\lceil\log n\rceil} 2^{j(\delta-\varepsilon)}=O\left(n^{1+\varepsilon}\right),
$$

since $\delta<\varepsilon$. The preprocessing time is also $O\left(n^{1+\varepsilon}\right)$.

4.2. Second data structure. Next, we describe the modified version of the second data structure, denoted as $\bar{\Psi}_{2}(\mathcal{P})$. We will construct a four-level partition tree. The first three levels of $\bar{\Psi}_{2}(\mathcal{P})$ will filter out the faces of polyhedra in $\mathcal{P}$ whose $x y$-projections contain an endpoint of $\gamma$, and the fourth level will determine whether any of these faces intersect the query segment.

In more detail, let $F$ be the set of faces of polyhedra in $\mathcal{P}$, and let $F^{*}$ denote the set of the $x y$-projections of these faces. By our assumption, each face in $F^{*}$ is a triangle. We split each face $f \in F$ into two subtriangles by drawing a plane parallel to the $y z$-plane through the "middle" vertex of $f$, as shown in Fig. 3. We will continue to denote the new set of faces by 


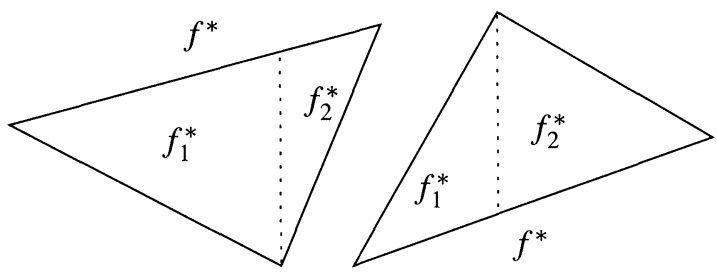

FIG. 3. Splitting each triangle into two triangles.

$F$ and the set of their $x y$-projections by $F^{*}$. Each triangle in $F^{*}$ has one vertical edge (i.e., parallel to the $y$-axis) and two nonvertical edges. Let $I$ denote the set of the $x$-projections of triangles in $F^{*}$. We construct a segment tree $B$ on $I$; see [30] for details on segment trees. Every node $v$ of $B$ is associated with an interval $\delta_{v}$ and stores a "canonical" subset $I(v)$ of $I$, where each interval in $I(v)$ contains $\delta_{v}$ (but does not contain $\delta_{p(v)}$, where $p(v)$ is the parent of $v)$. Moreover, $\sum_{v \in B}|I(v)|=O(n \log n)$. We preprocess each canonical subset separately.

Let $F^{*}(v)$ denote the set of triangles corresponding to the intervals in $I(v)$; put $t=$ $\left|F^{*}(v)\right|$. We construct a partition tree $\mathcal{T}=\mathcal{T}(v)$ on $F^{*}(v)$. For each triangle $\Delta \in F^{*}(v)$, pick one of its nonvertical edges. Let $V$ denote the set of points in the $x y$-plane, dual to the lines supporting these edges. Each node $w$ of $\mathcal{T}$ will be associated with a subset of $V$ and a triangle. The root $u$ is associated with $V$ and the entire $x y$-plane. Let $r$ be some appropriate constant. We construct, in linear time, a simplicial $r$-partition $\Pi=\left\{\left(V_{1}, \tau_{1}\right), \ldots,\left(V_{u}, \tau_{u}\right)\right\}$ for $V$ with crossing number $O(\sqrt{r})$ [25], [26]. We create a child $w_{i}$ of $u$ corresponding to each pair $\left(V_{i}, \tau_{i}\right)$ and store a two-level auxiliary structure at $w_{i}$, as detailed below. We recursively preprocess $V_{i}$ and attach the resulting tree of auxiliary substructures to $w_{i}$. The recursion stops when the number of points in $V_{i}$ falls below some prespecified constant.

The auxiliary structure at $w_{i}$ is constructed as follows. For each point $p \in V_{i}$, we pick the other nonvertical edge of the triangle corresponding to $p$. Let $W_{i}$ denote the set of points dual to lines supporting these edges; $\left|W_{i}\right| \leq\lceil t / r\rceil$. We construct a partition tree on $W_{i}$ as above. The root of the partition tree is associated with $W_{i}$ and the entire $x y$-plane. We compute a simplicial $r$-partition $\Pi_{i}=\left\{\left(W_{i 1}, \tau_{i 1}\right), \ldots,\left(W_{i u_{i}}, \tau_{i u_{i}}\right)\right\}$ for $W_{i}$, create a child $w_{i}$ of the root for each pair $\left(W_{i j}, \tau_{i j}\right)$, and recursively preprocess $W_{i j}$. Let $F_{i j}$ denote the set of faces in $F(v)$ corresponding to points in $W_{i j}$. If $F_{i j}$ contains two faces of the same polyhedron, we do not store any structure at $w_{i}$ (because, for any projected endpoint $p$ of a query segment, any set $F_{i j}$ that will be picked up by the query will have the property that all its projected triangles contain $p$, so an $F_{i j}$ of the above kind will never have to be processed by any query). Otherwise we preprocess $F_{i j}$ for segment-intersection detection queries in 3-space, using the algorithm of Agarwal and Matoušek [5], and store it at the node corresponding to $\left(W_{i j}, \tau_{i j}\right)$ as its auxiliary structure. This completes the description of the second data structure. Following the same analysis as for $\bar{\Psi}_{1}(\mathcal{P})$, one can show that the total space and preprocessing time required are $O\left(n^{1+\varepsilon}\right)$.

4.3. Answering a query. A segment-intersection detection query is answered exactly the same way as in $\S 3.3$. That is, we first query $\bar{\Psi}_{2}(\mathcal{P})$ and determine whether any of the faces, whose $x y$-projections contain an endpoint of $\gamma$, intersects $\gamma$. If $\gamma$ does not intersect any such face, then we determine, using $\bar{\Psi}_{1}(\mathcal{P})$, whether $e$ intersects any other face of the polyhedra in $\mathcal{P}$.

Let $p$ be the left endpoint of $\gamma^{*}$. We want to determine whether any face whose $x y$ projection contains $p$ intersects $\gamma$. A triangle $f^{*} \in F^{*}$ contains $p$ if and only if the following three conditions are satisfied: 
(i) the $x$-projection of $f^{*}$ contains the $x$-coordinate of $p$,

(ii) one of the nonvertical edges of $f^{*}$ lies above $p$, and

(iii) the other nonvertical edge of $f^{*}$ lies below $p$ (in the $x y$-plane).

To filter out the faces whose $x y$-projections satisfy these three conditions, we query the segment tree $B$ (the first-level structure of $\bar{\Psi}_{2}(\mathcal{P})$ ) with $p$ and compute the $O(\log n)$ nodes of $B$ whose associated intervals contain the $x$-coordinate of $p$. Let $v$ be such a node of $B$, and let $\ell$ denote the line dual to $p$. We query the (second-level) partition tree $\mathcal{T}=\mathcal{T}(v)$ with $\ell$. We start at the root and at each node $w$, visited by the algorithm, we do the following. Let $(V, \tau)$ be the pair associated with $w$. If $w$ is a leaf, we directly determine whether $\gamma$ intersects any of the triangles of $F$ corresponding to points in $V$. So assume that $w$ is an internal node. If $\tau$ intersects $\ell$, we visit all the children of $w$. Otherwise, we visit the auxiliary structure stored at $w$. Without loss of generality assume that $\tau$ lies fully above $\ell$; the other case can be handled symmetrically. We search the (third-level) auxiliary partition tree stored at $w$ with $\ell$ in the same way as we searched $\mathcal{T}(v)$. That is, at each node $\xi$ of this third-level structure, we do the following. Let $\left(W^{\prime}, \tau^{\prime}\right)$ be the pair associated with $\xi$. If $\ell$ intersects the triangle $\tau^{\prime}$, we recursively search at each child of $\xi$. If $\tau^{\prime}$ lies fully above $\ell$, then both nonvertical edges of triangles in $F_{\tau^{\prime}}^{*}$, the set of triangles of $F^{*}(v)$ corresponding to the points in $W^{\prime}$, lie above the left endpoint of $\gamma^{*}$, which implies that $p$ lies below all triangles in $F_{\tau^{\prime}}^{*}$. Consequently, we do not search the subtree rooted at $\xi$ any further. Finally, if $\tau^{\prime}$ lies fully below $\ell$, we can conclude that $p$ lies in the triangles of $F_{\tau^{\prime}}^{*}$. Let $F_{\tau^{\prime}} \subseteq F(v)$ denote the set of faces of polyhedra corresponding to triangles in $F_{\tau^{\prime}}^{*}$. Using the fourth-level auxiliary structure stored at $\xi$, we test whether $\gamma$ intersects any of the triangles in $F_{\tau^{\prime}}$. If $\gamma$ intersects a triangle of $F_{\tau^{\prime}}$, we stop right away, otherwise we continue with the overall search. If no face of $F(v)$, whose $x y$-projection contains $p$, intersects $\gamma$, we repeat the above step with the right endpoint of $\gamma$.

If the above procedure does not detect an intersection between $\mathcal{P}$ and $\gamma$, we query $\bar{\Psi}_{1}(\mathcal{P})$ as follows. We determine the segments of $E^{*}$ intersected by the $x y$-projection $\gamma^{*}$ of the query segment $\gamma$. Let $\Gamma$ be a canonical subset of the output to this subquery. We map the line containing $\gamma$ to its Plücker hyperplane $h$, and query the secondary structure (partition tree) constructed on the marked edges of $\Gamma$ with $h$. The root of the partition tree stores a simplicial $r$-partition $\Pi=\left\{\left(S_{1}, \triangle_{1}\right), \ldots,\left(S_{\alpha}, \triangle_{\alpha}\right)\right\}$. For each simplex $\triangle_{i}$ in $\Pi$, we test whether $h$ intersects $\triangle_{i}$. If $h$ intersects $\triangle_{i}$, we recursively search the substructure constructed on $S_{i}$. On the other hand, if $h$ does not intersect $\triangle_{i}$, all points in $S_{i}$ lie either above $h$ or below $h$, say above $h$. Then, as is easily checked, we know that all edges $e \in E\left(S_{i}\right)$ lie above $\gamma$, so it suffices to determine whether $\gamma$ intersects any of the faces incident to any edge in $E\left(S_{i}\right)$, or whether any edge of $\bar{U}_{i}$ lies below $\gamma$. Both of these conditions can be tested, by querying the third-level substructures stored with $E\left(S_{i}\right)$, in time $O\left(u^{3 / 4+\delta}+s \bar{u}^{1 / 2+\delta}\right)$ (where $u=\left|S_{i}\right|$ and $\left.s u \geq\left|\bar{U}_{i}\right|\right)$. This completes the description of the algorithm for answering a query.

We now analyze the total time spent in answering a query. First let us consider the time spent in querying $\bar{\Psi}_{2}(\mathcal{P})$. For $1 \leq i \leq 4$, let $Q^{(i)}(m, n)$ denote the maximum query time at an $i$ th level structure (including the time spent at its auxiliary structures), storing $n$ triangles which belong to $m$ different polyhedra. Since the above procedure visits only $O(\log n)$ nodes of the segment tree,

$$
Q^{(1)}(m, n)=O(\log n) \cdot Q^{(2)}(m, n) .
$$

The fourth-level structure of $\bar{\Psi}_{2}(\mathcal{P})$ has at most one triangle from each polyhedron, so $m=n$ and, by [5], $Q^{(4)}(m, n)=O\left(m^{3 / 4+\delta}\right)$, for any $\delta>0$. Finally, for $i=2,3$ (i.e., for partition trees constructed on sets of nonvertical edges of triangles), a line intersects only $O(\sqrt{r})$ triangles of the $r$-partition constructed on the set of points associated with any tree node. Therefore the query line recursively searches only $O(\sqrt{r})$ children of any interior node, which 
yields the following recurrence:

$$
Q^{(i)}(m, n) \leq \begin{cases}\sum_{i=1}^{c_{1} \sqrt{r}} Q^{(i)}\left(m_{i}, n_{i}\right)+Q^{(i+1)}(m, n) & \text { if } n \geq n_{0}, \\ O(1) & \text { if } n<n_{0},\end{cases}
$$

where $n_{0}, c_{1}$ are appropriate constants, and $n_{i} \leq n / r, m_{i} \leq m$ for each $i$. We claim that the solution of the above recurrence is

$$
Q^{(i)}(m, n) \leq A m^{1 / 4} n^{1 / 2+\varepsilon},
$$

for any $\varepsilon>0$. We will prove the recurrence for $i=3$; a similar proof works for $i=2$. Equation (6) is obviously true for $n \leq n_{0}$ (provided $A$ is chosen sufficiently large); and for $n>n_{0}$, we have, for an appropriate constant $c_{2}>0$,

$$
\begin{aligned}
Q^{(3)}(m, n) & \leq \sum_{i=1}^{c_{1} \sqrt{r}} Q^{(3)}\left(m_{i}, n_{i}\right)+c_{2} m^{3 / 4+\delta} \\
& \leq \sum_{i=1}^{c_{1} \sqrt{r}} A m_{i}^{1 / 4}\left(\frac{n}{r}\right)^{1 / 2+\varepsilon}+c_{2} m^{3 / 4+\delta} \\
& \leq A m^{1 / 4} n^{1 / 2+\varepsilon} \frac{c_{1}}{r^{\varepsilon}}+c_{2} m^{3 / 4+\delta} \\
& \leq A m^{1 / 4} n^{1 / 2+\varepsilon},
\end{aligned}
$$

provided that $\varepsilon \geq \delta$ and that the constants $r, A$ are chosen sufficiently large (we also use here the obvious fact that $m \leq n$ ).

Similarly, we can show that $Q^{(2)}(m, n)=O\left(m^{1 / 4} n^{1 / 2+\varepsilon}\right)$. Plugging (6) into (4), we can conclude that the maximum query of $\bar{\Psi}_{2}(\mathcal{P})$ is $O\left(m^{1 / 4} n^{1 / 2+\varepsilon}\right)$.

Next, we analyze the query time of $\bar{\Psi}_{1}(\mathcal{P})$. Recall that we spend $O\left(u^{3 / 4+\delta}+(s u)^{1 / 2+\delta}\right)$ time at a third-level substructure of size $u$. Let $Q^{(2)}(u)$ denote the maximum query time of the second-level partition constructed on a subset of $u$ marked edges of $\Gamma$. Since the crossing number of the simplicial $r$-partition stored at each node of the (second-level) partition tree is $O\left(r^{3 / 4} \log ^{5 / 4} r\right)$, we obtain the following recurrence:

$$
Q^{(2)}(u) \leq c_{1} r^{3 / 4} \log ^{5 / 4} r \cdot Q^{(2)}\left(\frac{u}{r}\right)+c_{2}\left(u^{3 / 4+\delta}+(s u)^{1 / 2+\delta}\right),
$$

where $c_{1}, c_{2}$ are appropriate constants. The solution of the above recurrence is easily checked to be

$$
Q^{(2)}(u)=O\left(s^{1 / 2+\delta} u^{3 / 4+\delta}\right) .
$$

Hence, the total time spent in querying a single first-level canonical subset $\Gamma$ is

$$
O\left(s^{1 / 2+\delta} \mu^{3 / 4+\delta}\right)=O\left(\left\lceil\frac{\nu}{\mu}\right\rceil^{1 / 2+\delta} \mu^{3 / 4+\delta}\right)=O\left(\mu^{1 / 4} v^{1 / 2+\delta}\right)
$$

Recall that there are $O\left(\left(n / 2^{j}\right)^{1+\varepsilon}\right)$ first-level canonical subsets of size between $2^{j-1}$ and $2^{j}$, so the overall query time of the first data structure is at most

$$
\sum_{j=1}^{\lceil\log n\rceil} O\left(\left(\frac{n}{2^{j}}\right)^{1+\varepsilon}\right) \cdot O\left(m^{1 / 4} 2^{j(1 / 2+\delta)}\right)=O\left(m^{1 / 4} n^{1 / 2+\varepsilon^{\prime}}\right),
$$


where $\varepsilon^{\prime} \geq \varepsilon+\delta$ is another arbitrarily small constant. Putting everything together, we obtain the following theorem.

THEOREM 4.2. Let $\mathcal{P}=\left\{P_{1}, \ldots, P_{m}\right\}$ be a collection of $m$ (possibly intersecting) convex polyhedra or polyhedral terrains in $\mathbb{R}^{3}$ with a total of $n$ edges. Given any $\varepsilon>0$, we can preprocess $\mathcal{P}$ in time $O\left(n^{1+\varepsilon}\right)$ into a data structure of size $O\left(n^{1+\varepsilon}\right)$, so that one can determine in time $O\left(m^{1 / 4} n^{1 / 2+\varepsilon}\right)$ whether a query segment intersects any polyhedron (or polyhedral terrain) in $\mathcal{P}$.

Again, we plug this procedure into the parametric search technique to answer a rayshooting query. We leave it to the reader to verify that each comparison can be reduced to determining whether the first intersection of the ray and $\mathcal{P}$ lies in a query half-space. Hence, we can conclude with the following theorem.

THEOREM 4.3. Let $\mathcal{P}=\left\{P_{1}, \ldots, P_{m}\right\}$ be a collection of $m$ (possibly intersecting) convex polyhedra or polyhedral terrains in $\mathbb{R}^{3}$ with a total of $n$ edges. Given any $\varepsilon>0$, we can preprocess $\mathcal{P}$ in time $O\left(n^{1+\varepsilon}\right)$ into a data structure of size $O\left(n^{1+\varepsilon}\right)$, so that the first intersection point, if any, of a query ray with the polyhedra of $\mathcal{P}$ can be computed in $O\left(m^{1 / 4} n^{1 / 2+\varepsilon}\right)$ time.

5. Application to motion planning. An interesting application of our algorithm for ray shooting amidst convex polyhedra is the following motion-planning problem in $\mathbb{R}^{3}$. Suppose we have a convex polyhedral object $B$ bounded by $k$ faces, which is free to translate amidst a collection of $m$ convex polyhedral obstacles, $A_{1}, \ldots, A_{m}$, with a total of $n$ faces. Preprocess them into a data structure so that, given any free placement $Z$ of $B$ and a direction $\mathbf{u}$, we can efficiently find the first obstacle, if any, to be hit as we translate $B$ from $Z$ in direction $\mathbf{u}$.

This problem can be easily reduced to the ray-shooting problem amidst a collection of intersecting convex polyhedral objects. We simply compute the Minkowski differences (also known as expanded obstacles)

$$
A_{i}^{*}=A_{i}-B_{0}=\left\{x-y \mid x \in A_{i}, y \in B_{0}\right\},
$$

for $i=1, \ldots, m$; here $B_{0}$ denotes some standard placement of the object $B$. If $A_{i}$ has $n_{i}$ faces, then $A_{i}^{*}$ is a convex polyhedron consisting of at most $O\left(k n_{i}\right)$ faces, so the total number of faces of the expanded obstacles is $O(\mathrm{kn})$.

Now, given a free placement $Z$ of $B$ and a direction $\mathbf{u}$, we can find the first obstacle to be hit by $B$ when it is translated from $Z$ in direction $\mathbf{u}$ by performing a ray-shooting query with the ray $(z, \mathbf{u})$ amidst the expanded obstacles $A_{i}^{*}$, where $z$ is the displacement of $B$ from its standard placement $B_{0}$ to the placement $Z$. The first expanded obstacle that the ray hits corresponds to the first obstacle that $B$ hits. Applying the results in the previous sections, we thus obtain the following corollary.

COROLLARY 5.1. Given a collection of $m$ convex polyhedral obstacles with a total of $n$ faces and a convex polyhedral object $B$ with $k$ faces, we can preprocess them, in time $O\left((k m n)^{2+\varepsilon}\right)$, into a data structure of size $O\left((k m n)^{2+\varepsilon}\right)$, so that, given any placement $Z$ of $B$ and direction $\mathbf{u}$, we can determine, in $O\left(\log ^{2} k n\right)$ time, the first obstacle, if any, that $B$ hits when translated from $Z$ in direction $\mathbf{u}$.

6. Conclusion. In this paper we presented two data structures for answering ray-shooting queries among a collection of $m$ convex polyhedra or polyhedral terrains with a total of $n$ faces. The first method answers a query in $O\left(\log ^{2} n\right)$ time using $O\left((m n)^{2+\varepsilon}\right)$ space and preprocessing time, while the second method achieves $O\left(m^{1 / 4} n^{1 / 2+\varepsilon}\right)$ query time, using $O\left(n^{1+\varepsilon}\right)$ space and preprocessing time. When $m \ll n$, both methods are significantly better than previous techniques, which either require $O\left(n^{4+\varepsilon}\right)$ space and preprocessing for a polylogarithmic query time, or require $O\left(n^{3 / 4+\varepsilon}\right)$ query time for almost-linear storage and preprocessing. Of course, when $m \approx n$, our algorithms perform as well as the previous ones. For $m=1$, the performance 
of our algorithms matches that of the best known algorithm for ray shooting in a polyhedral terrain [4], [15], but is not as good as the best known technique for a single convex polyhedron.

We conclude by mentioning some open problems:

1. If $m=1$, neither of our structures achieves close-to-optimal performance for the case of a single convex polyhedron. For example, the space and preprocessing time of our first technique are $O\left(n^{2+\varepsilon}\right)$, in contrast with the technique of [20], which can answer a ray-shooting query in $O(\log n)$ time, using $O(n)$ space and $O(n \log n)$ preprocessing. It is clear from this that we are not fully exploiting the fact that the given polyhedra are convex. It would be interesting to improve our techniques further so that their performance approaches that of [20] for the case of a single convex polyhedron. (We are also not exploiting at all the pairwise disjointness of the given polyhedra.) A plausible goal to shoot for might be to improve our first technique so that it yields a data structure that requires only $O\left(m^{4+\varepsilon}+n\right)$ or $O\left(m^{3+\varepsilon} n\right)$ space and preprocessing.

2. How far can our techniques be extended? It seems unlikely that any improvement over the general previous techniques can be obtained for ray shooting among arbitrary nonconvex polyhedra, but perhaps there are useful special cases, beyond the case of terrains, for which faster techniques exist.

3. One application of our algorithms is for the case of a small number of curved surfaces, each approximated by a polyhedral surface with a large number of faces. An alternative attack on this case would be to drop the polyhedral representation altogether and to develop special techniques for ray shooting amidst curved objects. This is a more difficult problem for general surfaces, although some progress has recently been done for the case of ray shooting amidst spheres (see [2] and [5]).

4. Finally, as mentioned in the introduction, no nontrivial lower bounds are known for any of the ray-shooting problems.

Acknowledgments. The authors thank Mark de Berg and other attendants of the 2nd Utrecht Workshop on Computational Geometry and Its Application for raising the problem studied here.

\section{REFERENCES}

[1] P. K. AgARwal, Ray shooting and other applications of spanning trees with low stabbing number, SIAM J. Comput., 21 (1992), pp. 540-570.

[2] P. K. Agarwal, L. Guibas, M. Pellegrini, and M. Sharir, manuscript, 1993.

[3] P. K. Agarwal, M. van Kreveld, and M. Overmars, Intersection queries for curved objects, J. Algorithms, 15 (1993), pp. 229-266.

[4] P. K. Agarwal and J. MatoušEK, Ray shooting and parametric search, SIAM J. Comput., 22 (1993), pp. 794-806.

[5] - Range searching with semi-algebraic sets, Discrete Comput. Geom., 11 (1994), pp. 393-418.

[6] P. K. Agarwal And M. Sharir, Applications of a new space partitioning technique, Discrete Comput. Geom., 9 (1993), pp. 11-38.

[7] ——, Ray shooting amidst convex polygons in 2D, J. Algorithms, to appear.

[8] B. Aronov, M. Pellegrini, AND M. Sharir, On the zone of a surface in a hyperplane arrangement, Discrete Comput. Geom., 9 (1993), pp. 177-188.

[9] R. BAR YehUdA AND S. Fogel, Variations on ray shooting, Algorithmica, 11 (1994), pp. 133-145.

[10] M. De Berg, D. Halperin, M. Overmars, J. Snoeyink, AND M. Van Kreveld, Efficient ray shooting and hidden surface removal, Algorithmica, 12 (1994), pp. 30-53.

[11] B. Chazelle, Cutting hyperplanes for divide-and-conquer, Discrete Comput. Geom., 10 (1993), pp. $145-158$.

[12] - Lower bounds on the complexity of polytope range searching, J. Amer. Math. Soc., 2 (1989), pp. 637-666.

[13] B. Chazelle And H. Edelsbrunner, An optimal algorithm for intersecting line segments in the plane, J. ACM, 39 (1992), pp. 1-54.

[14] B. Chazelle, H. Edelsbrunner, M. Grigni, L. Guibas, J. Hershberger, M. Sharir, and J. Snoeyink, Ray shooting in polygons using geodesic triangulations, Algorithmica, 12 (1994), pp. 54-68. 
[15] B. Chazelle, H. Edelsbrunner, L. Guibas, M. Sharir, And J. STolfi, Lines in space: Combinatorics and algorithms, Tech. rep. 491, Dept. of Computer Science, New York University, February 1990; Algorithmica, to appear.

[16] B. Chazelle and J. Friedman, A deterministic view of random sampling and its use in geometry, Combinatorica, 10 (1990), pp. 229-249.

[17] B. Chazelle AND L. Guibas, Visibility and intersection problems in plane geometry, Discrete Comput. Geom., 4 (1989), pp. 551-589.

[18] B. Chazelle, M. Sharir, AND E. Welzl, Quasi-optimal upper bounds for simplex range searching and new zone theorems, Algorithmica, 8 (1992), pp. 407-430.

[19] K. L. ClaRKSON, A randomized algorithm for closest-point queries, SIAM J. Comput., 17 (1988), pp. 830-847.

[20] D. DOBKIN AND D. KIRKPATRICK, Determining the separation of preprocessed polyhedra: A unified approach, Proc. 17th Internat. Colloq. Automata, Languages and Programming, 1991, pp. 400-413.

[21] L. Guibas, M. OVERMARS, AND M. SharIR, Ray shooting, implicit point location, and related queries in arrangements of segments, Tech. Rep. 433, Dept. of Computer Science, New York University, March 1989.

[22] J. HERSHBERGER AND S. SURI, A pedestrian approach to ray shooting: Shoot a ray, take a walk, J. Algorithms, 18 (1995), pp. 403-431.

[23] J. MATOUŠEK, Approximations and optimal geometric divide-and-conquer, J. Comput. System Sci., 50 (1995), pp. 203-208

[24] - Reporting points in halfspaces, Computational Geometry: Theory and Applications, 2 (1992), pp. 169-186.

[25] - Efficient partition trees, Discrete Comput. Geom., 8 (1992), pp. 315-334.

[26] - Range searching with efficient hierarchical cuttings, Discrete Comput. Geom., 10 (1993), pp. 157-182.

[27] K. Mehlhorn, Data Structures and Algorithms, III. Multi-Dimensional Searching and Computational Geometry, Springer-Verlag, Berlin, Heidelberg, New York, 1985.

[28] M. Pellegrini, Ray shooting in triangles in 3-space, Algorithmica, 9 (1993), pp. 471-494.

[29] M. Pellegrini AND P. Shor, Finding stabbing lines in 3-space, Discrete Comput. Geom., 8 (1992), pp. 191-208.

[30] F. Preparata and M. Shamos, Computational Geometry: An Introduction, Springer-Verlag, Heidelberg, 1985.

[31] D. M. H. Sommerville, Analytical Geometry in Three Dimensions, Cambridge University Press, Cambridge, UK, 1951. 